\title{
Received and Perceived Status of Health Management Information System (HMIS) Software: A Structural Equation Model (SEM) Approach
}

\author{
C. Sriram, V. Mohanasundaram \\ ${ }^{1}$ Assistant Professor, Department of Visual Communication, Dwaraka Doss Goverdhan Doss Vaishnav College, \\ Chennai, Tamil Nadu, India, ${ }^{2}$ Associate Professor and Head, Department of Economics (UA), PSG College of Arts \\ \& Science, Coimbatore, Tamil Nadu, India
}

\begin{abstract}
More thansupply the demand for health care usually determines the design of public health services. This is more so when technological developments, including that of information and communication technologies, pierce through the process of utilization of any service by the public. Although prior research papers have focused on the factors that impact on the adoptionof information technology, there are limited empirical research works that simultaneouslycapture technology factors (TAM, TAM2) and end-user development specific factors (perceived technology usage and intention to recommend) helping healthcare professionals to adopt Health Management Information System (HMIS) software in the healthcare environment. To fill this gap, the present paper used the Technology Acceptance Model (TAM), the extended TAM model (TAM2) and identified the important determinants of user acceptance perceived risk and trust. This is specifically undertaken in order to describe ESIChealthcare professionals behavioral intention to adopt HMIS software services. The study was conducted in the Employees' State Insurance Corporation (ESIC) main hospital and dispensaries in the Tirunelveli sub-region. The required data were collected from 171 ESIC healthcare professionals in the Tirunelveli sub-region. AStructural Equation Model (SEM) approach was used. Convergence anddivergence with earlier findings were found, confirming that Perceived Usefulness (PU), Perceived Ease of Use (PEOU), social influence, facilitating conditions and training hadsignificant influence on the intention of healthcare professionals to adopt HMIS software. The study provideda basis for further refinement of technology adoption model.Improvingperceived usefulness factor (perceived long-term usefulness) may turn healthcare professionals towards adoption of HMIS.
\end{abstract}

Keywords: Information Technology, Technology Acceptance Model, Employees' State Insurance Corporation, Perceived Usefulness and Perceived Ease of Use.

\section{Introduction}

In recent years, there has been growing global emphasis on the need for Information Systems (IS)

\section{Corresponding Author:}

\section{Dr. C. Sriram}

Assistant Professor, Department of Visual Communication, Dwaraka Doss Goverdhan Doss Vaishnav college, Chennai, Tamil Nadu Address: 8/7 $1^{\text {st }}$ street Jai nagar ( $2^{\text {nd }}$ floor), Arumbakkam, Chennai-600106, Tamil Nadu, India e-mail: srirammediaguy@gmail.com in all sectors. The elementary conditions that enable global competition in the information society are the development of communication technologies and the importance of the administrative information flows and the inter-institutional communication networks. Therefore, more emphasis is placed on flexible and cost efficient information systems in the competitive presentation of public services. Information systems are one of the means by which public institutions can save economic indicators, such as time and cost. Assuring and promoting quality in health care services continues to be a priority for any health care system. Besides evaluationof health status through morbidity andmortality estimates, there has been equalemphasis 
on quality of care indicators inhealth systems research. The continuously increasing demand for assessment of quality in thehealth care system can be attributed to risingcosts, constrained resources and evidenceof variations in clinical practice.Hospital management information systems provide an institutional framework consisting of different information about the medical, financial and managerial functions of a particular hospital. The first applications of these systems are limited to, the recording of patient information and the billing of health services offered. Today, new modules have been added to health management information systems such as appointment over the internet, follow-up patients, and request analysis and display results. Thus, it has become possible for doctors to transmit their requests directly to the laboratories via automation systems and to monitor the results online. In this respect, the main purpose of the study is to define the role of hospital management information system within the functional context. Further, it is crucial to analyze the genuine perception of healthcare professionals' willingness to adopt these technologies. In order to identify which factors influence on healthcare professional's intention to adopt HMIS the researcher has merged theexisting and empirically validated theoretical models (TAM and TAM2) with perceived benefitsand actual use of technology. The study uses constructs from the Technology Acceptance Model (TAM) and the extended Technology Acceptance Model (TAM 2) and integrates the constructs of facilitating conditions and training in the model. Thus, cognitive factors, perceived social forces and intrinsic characteristics are also included in a unified model along with facilitating conditions and training. The study measures intention-to-use health management information system software in the ESIC healthcare environment. Intention to use has been found to be a strong predictor of actual system usage in the available literature. The study uses a quantitative survey method to record ESIC healthcare professionals' opinions and attitudes. It analyzes the data using a refinement procedure, controlling reliability and validity, and validates the proposed model using Structural Equation Modeling.

Review of Literature: IT stress is said to be an important factor and its evaluation techniques are based on the widely used Technology Acceptance Model (TAM). IT stress could be broadly classified into two types. They were Direct IT stress and Indirect IT stress (Raitoharju, 2005). Direct IT stress has always been a hindrance and is caused by the following factors: person's fear of breaking something, feeling of ignorance, fear of technology, fear about health, fear about anything new and unfamiliar or sense of threat to intellectual selfassessment. Indirect stress is all about time consumption and pressure. ${ }^{1}$ Here, the user is an expert in information technology and spends a lot of time in IT related works. Since he spends a lot of time in front of computers, he is unable tocomplete other tasks properly. This creates unwanted pressure and such a pressure gives way to indirect IT stress. Further, computer anxiety reduced the effectiveness of computerization. Perceived usefulness and Perceived ease of use played an important role in IT acceptance studies. ${ }^{7}$ Perceived usefulness encouraged IT usage and thereby resulted in technology acceptance. However, Perceived ease of use did not have any significant effect on Perceived usefulness. ${ }^{3}$ While it is important to look at systemic, organizational and professional factors that shape the integration of ICT in health care systems, studying adoption at the individual level is particularly relevant since the ultimate decision to use a new technology is often that of the individual (Hu et al., 2001; Igbaria et al., 1997).

Health Information System (HIS) could be categorized by their complexity and integrity. The HIS could integrate a large variety of medical and administrative information and further, low quality information could have dramatic repercussions on patient healthcare (Ribière, J.LaSalle, Khorramshahgol, \& Gousty, 1999). HIS quality influences the quality of care by capturing, transferring, storing, managing and displaying medical information. Efficient medical decision making, customer satisfaction and reduced health related risks are found to be the prime goals of health information system. ${ }^{\mathbf{8}}$ System Quality, Information Quality, Use, User Satisfaction, Individual Impact and Organizational Impact were found to be the important dimensions that come under quality of information system. Moreover, adaptation to common tasks and user friendliness could enable increased levels of performance.

Information Technology (IT) increased quality of care indirectly by improving both relational and management continuity of care. Information Technology, through electronic integration, contributed to relational and management continuity as well as to improving quality of care (Pinsonneault, Dakshinamoorthy, Reidel, \& Tamblyn, 2012). IT systems should be designed and implemented in such a way so as to motivate patients to 
visit a given physician or at least physicians who share important patient record information. The quality of care could be further improved when physicians were electronically integrated with other specialists, retail pharmacies and the health insurance agency. ${ }^{6}$

The sustainability of Health Information System depended on the technical features and physical infrastructures. Any lack of technical approach would lead to instability of the information system. Sociotechnical aspects played an all important role in information system. Integration of such aspects into the organizational structures would lead to stability (Kimaro \& Nhampossab, 2007). They would also enable easy execution of routine activities. Health services mainly include curative and preventive activities. These activities are hampered by scarce resources. The main aim of HIS is to overcome the scarcity and bring about better care. ${ }^{4}$ Further, extra attention should be given to users' needs, evaluation and feedback generation and future improvements.Information generation and sharing of knowledge within the sustainability strategy framework would also add to the stability of health systems.

Large health care infrastructures would always be confronted by the presence of the World Wide Web and electronic commerce. Community Health Information Networks (CHINs) have been defined as: Inter organizational systems (IOS) using information technology(ies) and telecommunications to store, transmit, and transform clinical and financial information. ${ }^{5}$ This information can be shared among cooperative and competitive participants, such as payers, hospitals, alternative delivery systems, clinics, physicians, and home health agencies. Customers (patients) were often described as 'by-products' of the health care delivery system. Economic dimensions, such as reduced risk, lower transaction costs and increased industry knowledge orcompetencies, were usually considered prior to the adoption stage of implementation (Payton, 2000).

The decomposed TPB model was advantageous to explain physicians' adoption and intentions to use the Information system (IS). The three antecedent constructs used in the TPB model were attitude, Subjective norm (SN), and perceived behavioral control (PBC). While attitude was decomposed into perceived usefulness (PU) and perceived ease of use (PEOU), PBC was decomposed into personal innovativeness in IT (PIIT), self-efficacy, and facilitating conditions. Perceived usefulness and perceived ease of use had significant impact on attitude of the physicians; perceived ease of use had significant impact on perceived usefulness. Further, Subjective norm could be predicted by interpersonal influence (Hung, $\mathrm{Ku}, \&$ Chien, 2008). Facilitating conditions is defined as the beliefs about availability of resources to facilitate that behavior. The facilitating conditions had no significant effect on perceived behavioral control. ${ }^{2}$ This is because, the information infrastructure (eg) internet is already available to most organizations and hence the influence of the facilitating conditions on perceived behavior control gradually decreases.

Hypotheses Formulation: This paper proposes a model, which unifies (a) cognitive factors or cognitive instrumental processes, such as job duties, output quality; (b) perceived social forces or social influence processes, such as subjective norm, voluntariness and image; (c) intrinsic characteristics of innovations compatibility, relative advantage, along with (d) computer selfefficacy; and (e) facilitating conditions. Consequently, in the unified model, the following hypotheses are tested:

H1a System Quality may positively impact the perceived usefulness of HMIS.

H1b System Quality may positively impact the perceived ease of use of HMIS.

H1c System Quality may positively impact the perceived behavioral control of HMIS users.

H2a Computer self-efficacy may positively impact the perceived usefulness of HMIS.

H2b Computer self-efficacy may positively impact the perceived ease of use of HMIS.

H2c Computer self-efficacy may positively impact the perceived behavioral control of HMIS users.

H3a Facilitating conditions may positively impact the perceived behavioral control of HMIS users.

H3b Facilitating conditions may positively impact the computer self-efficacy of HMIS users.

H4 Users' perceived behavioral control may positively impact the intention of HMIS adoption.

H5a HMIS perceived ease of use may positively impact the perceived usefulness of HMIS. 
H5b HMIS perceived ease of use may positively impact attitudes toward HMIS adoption.

H6a HMIS perceived usefulness may positively impact attitudes toward HMIS adoption.

H6b HMIS perceived usefulness may positively impact the intention to adopt HMIS.

H7 Attitudes toward adopting HMIS may positively impact the intention of adopting HMIS.

H8 HMIS subjective norms may positively impact the intention of adopting HMIS.

H9Attitudes towards adopting HMIS may positively impact the perceived behavioral control of HMIS users.

\section{Methodology}

An empirical research study was conducted using a research questionnaire with close-ended questions. Healthcare professionals in the ESIC main hospital and dispensaries in Tirunelveli sub-region have been chosen to be surveyed. The ESIC healthcare professionals were selected using convenience sampling. Among the ESIC hospital staff, there were 171 healthcare professionals in the Tirunelveli sub-region and all the 171 professionals in different departments of the hospital were selected for the study. The questionnaire used in this study was adopted from previous studies. Five point Likert scales were used, ranging from strongly agree to strongly disagree. The questionnaire consisted of 10 parts:

(1) General Information; (2) General System Quality; (3) Computer Self-efficacy; (4) Facilitating conditions; (5)Perceived Usefulness (PU); (6) Perceived Ease of Use (PEOU); (7) Perceived Behavioral Control (PBC); (8) Attitude; (9) Subjective norm (social influence); (10) Behavioral Intention (BI).

A pilot study using an extended questionnaire containing all the scales proposed in the literature review was conducted by administering the questionnaire to 25 ESIC healthcare professionals. Scales and items used in the questionnaire and the analysis have been successfully used in previous studies to measure information system adoption by the healthcare professionals in the hospital environment. These particular scales may not be adequate for measuring attitudes of the particular target group of ESIC healthcare professionals. However, reliability and validity analysis performed hereafter proves that the scales constitute a reliable and valid instrument for measuring information system adoption by ESIC healthcare professionals. For statistical analysis, Structural Equation Model (SEM) approach was used to identify the influential factors to ESIC healthcare professionals' acceptance of HMIS software in the hospital environment.

\section{Data and Analysis:}

Table 1: Model Summary (Goodness of fit Index)

\begin{tabular}{|c|c|c|c|c|c|c|}
\hline Model & RMR & RMSEA & GFI & AGFI & NFI & CFI \\
\hline & .074 & .061 & .968 & .945 & .973 & .946 \\
\hline
\end{tabular}

Note. NFI = Normed-fit index; GFI = Goodness-of-fit index; AGFI = adjusted goodness-of-fit index; RMR=Root Mean Square Residuals; RMSEA= Root Mean Square Error of Approximation; CFI=Comparative fit index.

The above table shows the model fit indices for the overall structural model of behavioral intention. The table reveals that the Root Mean Square Error of Approximation (RMSEA) value 0.61 and Root Mean Square Residuals (RMR) .074 which is lesser than equal to .08 (i.e. the model is mediocre fit (Hans Muller,2003), the other model fit evaluation method such as Goodness of fit index (GFI) value .968 , Adjusted Goodness of fit index (AGFI) value .945, Normed Fit Index (NFI) value .973 and Comparative fit index (CFI) value .946 are attained and satisfied the criteria (thumb rule i.e. greater than equal to .90). It is concluded that items perfectly measures behavioral intention model (i.e. an acceptable fit).

This section presents results of hypotheses testing. Table shows sixteen hypotheses represented by causal paths that were used to test the relationships between the latent constructs. The latent constructs used in the proposed theoretical model were classified in two main categories: exogenous and endogenous constructs. Exogenous constructs were the Facilitating conditions 
(p4_tot) and System Quality (p2_tot) whileEndogenous constructs were the Computer Self efficacy(p3_tot), Perceived Usefulness (p5_tot), Perceived ease of use (p6_tot), Perceived Behavioral Control (p7_tot), Attitude (p8_tot), Subjective norm (p9_tot), Behavioral Intention ( $\mathrm{p} 10 \_$tot). Goodness of fit indices and other parameters estimates were examined to evaluate the hypothesized structural model. Assessment of parameter estimates results suggested that sixteen hypothesized paths were significant. Thus, indicating support for the sixteen hypotheses. These results are presented in detail as follows.

Table 2: Standardized Regression Weights: (Group number 1 - Default model)

\begin{tabular}{|c|c|c|c|c|c|}
\hline \multicolumn{3}{|c|}{ Variables } & \multirow{2}{*}{$\begin{array}{c}\text { Estimate } \\
.739\end{array}$} & \multirow{2}{*}{$\frac{\text { Hypotheses }}{\mathrm{H} 3 \mathrm{~b}}$} & \multirow[b]{2}{*}{ Facilitating conditions and Self efficacy } \\
\hline p3_tot & $<---$ & p4_tot & & & \\
\hline p5_tot & $<---$ & p2_tot & .555 & H1a & System Quality and Perceived Usefulness \\
\hline p6_tot & $<---$ & p2_tot & .299 & $\mathrm{H} 1 \mathrm{~b}$ & System Quality and Perceived ease of Use \\
\hline p5_tot & $<---$ & p3_tot & .280 & $\mathrm{H} 2 \mathrm{a}$ & Self efficacy and Perceived Usefulness \\
\hline p6_tot & $<---$ & p3_tot & .389 & $\mathrm{H} 2 \mathrm{~b}$ & Self efficacy and Perceived ease of use \\
\hline p8_tot & $<---$ & p5_tot & .508 & H6a & Perceived usefulness and Attitude \\
\hline p8_tot & $<---$ & p6_tot & .249 & $\mathrm{H} 5 \mathrm{~b}$ & Perceived ease of use and Attitude \\
\hline p7_tot & $<---$ & p2_tot & .287 & $\mathrm{H} 1 \mathrm{c}$ & System Quality and Perceived behavioral Control \\
\hline p7_tot & $<---$ & p3_tot & .246 & $\mathrm{H} 2 \mathrm{c}$ & Self efficacy and Perceived Behavioral Control \\
\hline p7_tot & $<---$ & p4_tot & .395 & $\mathrm{H} 3 \mathrm{a}$ & Facilitating Conditions and Perceived Behavioral Control \\
\hline p9_tot & $<---$ & p8_tot & 1.044 & H9 & Attitude and Subjective Norm \\
\hline p10_tot & $<---$ & p8_tot & .187 & $\mathrm{H} 7$ & Attitude and Behavioral Intention \\
\hline p10_tot & $<---$ & p9_tot & .294 & $\mathrm{H} 8$ & Subjective norm and Behavioral Intention \\
\hline p10_tot & $<--$ & p7_tot & .192 & $\mathrm{H} 4$ & Perceived Behavioral Control and Behavioral Intention \\
\hline p10_tot & $<---$ & p5_tot & .227 & $\mathrm{H} 6 \mathrm{~b}$ & Perceived Usefulness and Behavioral Intention \\
\hline
\end{tabular}

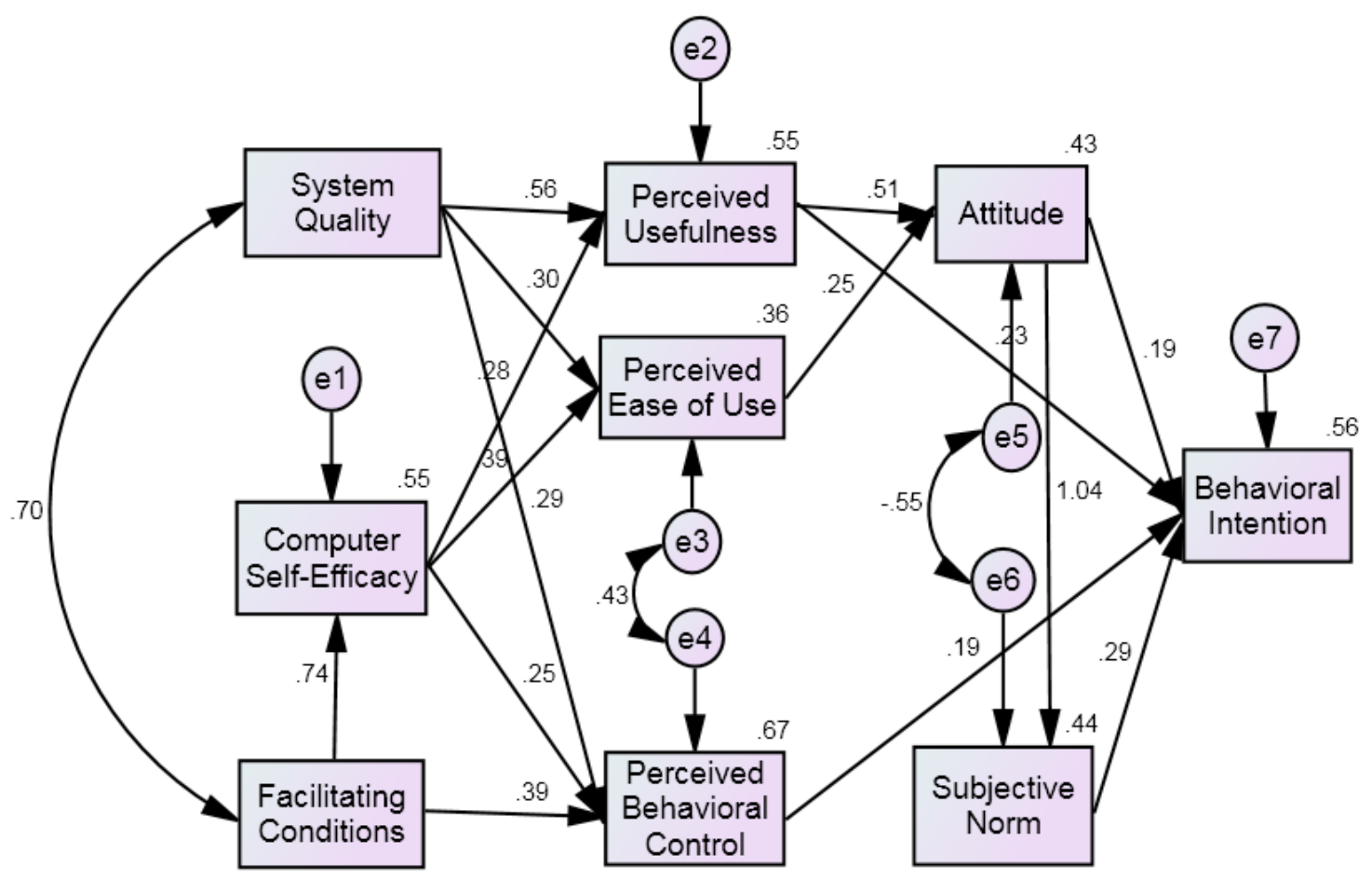

Figure 1: The research model after validation 
The table depicts the standardized regression weights $(\beta)$ which estimate and predict the intention of ESIC healthcare professionals towards HMIS adoption. The SEM result reveals that the hypotheses Self efficacy_ Perceived Usefulness (H2a), Self efficacy_ Perceived Ease of Use (H2b) and Self efficacy_ Perceived Behavioral Control $(\mathrm{H} 2 \mathrm{c})$ were significant. The standardized regression weights of $\mathrm{H} 2 \mathrm{a}(.280)$, $\mathrm{H} 2 \mathrm{~b}(.389)$ and H2c (.246), show that Self efficacy has a direct significant effect on Perceived Usefulness (28\%), Perceived Ease of Use (38.9\%) and Perceived Behavioral Control (24.6\%).

The hypotheses System Quality_ Perceived Usefulness (H1a), System Quality_ Perceived Ease of Use (H1b), System Quality_Perceived Behavioral Control (H1c) and Facilitating Condition_ Perceived Behavioral Control (H3a) were significant. The standardized regression weights of H1a (.555), H1b (.299), and H1c (.287), show that System Quality has a direct significant effect on Perceived Usefulness (55.5\%), Perceived Ease of Use (29.9\%) and Perceived Behavioral Control (28.7\%). It also reveals facilitating conditions has a significant direct effect on the Perceived Behavioral control(39.9\%) andSelf efficacy (73.9\%).

The hypotheses Perceived Usefulness_ Attitude (H6a), Perceived Ease of Use_Attitude (H5b) and Subjective Norm _ Attitude (H9) were significant. The standardized regression weights of H6a (.580), H5b (.249) and H9show that Perceived Usefulness (58\%), Perceived Ease Use (24.9\%) and Subjective Norm (104.4\%) have a direct significant effect on Attitude of ESIC healthcare professionals.

The hypotheses Perceived Usefulness_Behavioral Intention (H6b), Perceived Ease of Use_ Behavioral Intention (H4), Attitude_Behavioral Intention (H7) and Subjective Norm_ Behavioral Intention (H8) were also significant. The standardized regression weights of $\mathrm{H} 6 \mathrm{~b}$ (.227), H4 (.192), H7 (.187) and H8 (.294) show that Perceived Usefulness (22.7\%), Perceived Ease of Use (19.2\%), Attitude (18.7\%) and Subjective Norm (29.4\%) have a significant direct effect on Behavioral Intention of ESIC healthcare professionals.

\section{Discussion}

The results of this study provide support for the research model presented in Fig.1 and regardinghypotheses directional linkage. Findings from the present study suggest that in the presence of both facilitating variables (Computer Self-efficacy, Facilitating Conditions) and operational variables (Perceived Usefulness, Perceived Ease of Use) it is the second set that has a significant effect on intention to use. In more detail: System Quality, Attitude and Subjective Norm, along with Perceived Usefulness and Perceived Ease of Use are the five factors that influence intention to use HMIS. Computer Selfefficacy and Facilitating Conditions, although included in the model, do not significantly affect intention to use.Previously researchers have focused on the factors that impact on theadoption of health information technology; there is a limited empirical research work that simultaneouslycaptures technology factors and user specific factors that help healthcare professional'sadopt HMIS software. Thus, the study has aimed to develop an integrated technology adoption model with TAM and TAM2 model to predict and explain ESIC healthcare professional's intention to adopt HMIS software andrecommend HMIS in ESIC hospital environment. Convergence and divergence with earlierfindings were found, confirming that Perceived Usefulness, Perceived ease of Use and social influence (subjective norms) have significant influence in user's intention to adopt HMIS and these findings are consistentwith previous study conducted by Raitoharju (2005). Contrary to the expectation the researcher has found thatfacilitating condition and computer self-efficacy with intention to adopt HMIS were notvalid, these findings are consistent with Hung, $\mathrm{Ku}$ and Chien (2008). What the findings of this paper suggest is that along with emphasizing and augmenting technological benefits of using HMIS services, a greater and primary effort should be made in providing efficient and effective HMIS practices. These could satisfy ESIC healthcare professionals and engage them to use HMIS services further. Raising system quality and enhanced training sessions are important steps, but providing results and efficient solutions to ESIC healthcare professionals through HMIS software is more important in raising their intentions to use them. Users find health information systems useful when they relate to their intrinsic characteristics, their interest and their working settings. Users will use health management information systems if they help them to work more efficiently and effectively.

\section{Conclusion}

This study has limitations that provide the impetus for further research in this field of investigation.First, this research is cross sectional and measures the ESIC 
healthcare professional's intention at one point intime that may be less generalizable as compared with longitudinal study. Second, testing of this newly developed integrated technology model in other developingcountries may be useful for the further generalization of this model. The paper made a contribution to the study of adoption of health management information system by applying a model which joins TAM and TAM2. The effort was to measure the contribution of the separate variables in the presence of a multitude of effects.Thus, it is clear from the findings that for the adoption of HMIS by ESIC healthcare professionals, practical and operational issues regarding the efficiency of services should be considered first. This study can serve as a starting point for other health information system adoption researches. The user has to be placed at the center of future developments, thus, the board of members in the governing body of the hospital should take into consideration user needs, established marketing practices to promote the services and provide training to healthcare professionals in order to make the systems attractive to them. Training programs should stress the potential of health information systems and should help users to understand the relative advantage, job relevance and generally the value of them over existing manual systems. The findings can also provide useful recommendations to the development of practices and policies that influence intention to use HMIS services.

Acknowledgement: The authors express their gratitude and thanks towards all who have directly orindirectly helped them to complete this study.

Conflicts of Interest Disclosure: The authors declare that there is no conflict of interest statement.

Source of Funding: This is a self-funded study.

Ethical Clearance: The ethical approval was taken from ethical committee of Bharathiar university, Coimbatore, Tamil Nadu. Ethical clearance was obtained from the university.

\section{References}

1. $\mathrm{Hu} \mathrm{PJ}$, et al. Investigating technology implementation in a neurosurgical teleconsultation program: A case study inHong Kong. Proceedings of the 34th Hawaii International Conference on System Sciences, IEEE Computer Society. 2001.

2. Hung $\mathrm{S} \mathrm{Y}, \mathrm{Ku}$ Y C, \& Chien J C. Re-examining factors influencing information technology acceptance by individual professionals. PACIS. 2008.169.

3. Igbaria $\mathrm{M}$ et al. Personal computing acceptance factors in small firms: A structural equation model. MISQuarterly. 1997;21(3):279-302.

4. Kimaro H C, \& Nhampossab J L. The challenges of sustainability of health information systems in developing countries: comparative case studies of Mozambique and Tanzania. Journal of Health Informatics in DevelopingCountries.2007:1-10.

5. Payton F.C. Lessons learned from three interorganizational health care information systems. Information \& Management Journal. 2000:311-21.

6. Pinsonneault A, Dakshinamoorthy V, Reidel K, \& Tamblyn R. The impact of IT on quality of care: Evaluation of an integrated chronic disease management system. 45th Hawaii International Conference on System Sciences. Hawaii: IEEE Computer Society.2012:2947-56.

7. Raitoharju R. When acceptance is not enough taking TAM-model intohealthcare. Proceedings of the 38th Hawaii International Conference on SystemSciences. Hawaii: IEEE.2005:1-6.

8. Ribière V, J La Salle A, Khorramshahgol R, \& Gousty Y. Hospital Information Systems Quality: A Customer Satisfaction Assessment Tool. Proceedings of the 32nd Hawaii International Conference on System Sciences. Hawaii: IEEE. 1999:1-9. 